\title{
Correlation between heavy flavour production and multiplicity in string fusion approach
}

\author{
Vladimir Kovalenko ${ }^{1}$ and Vladimir Vechernin ${ }^{1, a}$ \\ ${ }^{1}$ Saint Petersburg State University, 7/9 Universitetskaya nab., St. Petersburg, 199034 Russia.
}

\begin{abstract}
The multiplicity dependence of heavy flavour production in pp-collisions at LHC energies is studied in the framework of string fusion approach. We applied a Monte Carlo model [1,2], with the string configurations simulating event-by-event. We assumed that the heavy flavour yield is proportional to the number of initial strings, whereas a total charged particle multiplicity is influenced by the string fusion process and can be obtained according to the standard string fusion prescriptions. We show that under this assumption the faster-than-linear growth of the open charm production, observed in experiment [3], can be related to the reduction of the total multiplicity due to string overlapping and fusion. The influence of this effect on forward-backward correlations involving heavy flavours is also discussed.
\end{abstract}

\section{Introduction}

The recent measurements on multiplicity dependence of the heavy quark production, performed by the ALICE Collaboration [3], demonstrated unexpected results. It was obtained that the D meson yield $N_{\mathrm{D}}$, plotted as a function of charged multiplicity $N_{\mathrm{ch}}$ in relative variables, shows faster-than-linear behaviour.

This data have been interpreted in EPOS 3 model [4] with the hydrodynamical stage of the evolution, and a simple string percolation model [5]. In both models, the growth of charmed yield faster than $N_{\text {ch }}$ is related to some physical process, which dumps multiplicity of bulk particles, but has almost no effect on $\mathrm{c}-\overline{\mathrm{c}}$ pair production.

We should note, that in the simple string percolation model [5] it is assumed that the string density is the same for every p-p collision and doesn't fluctuate. In the present report, we performed the calculations in more realistic Monte Carlo model with string fusion [1,2], where the string configurations are simulated event-by-event.

\section{The model}

The present model $[1,2]$ is based on the partonic picture of pp-scattering. The partons are distributed in transverse plane with Gauss distribution relative to the center of nucleon and arranged into dipoles.

ae-mail: v.vechernin@spbu.ru 


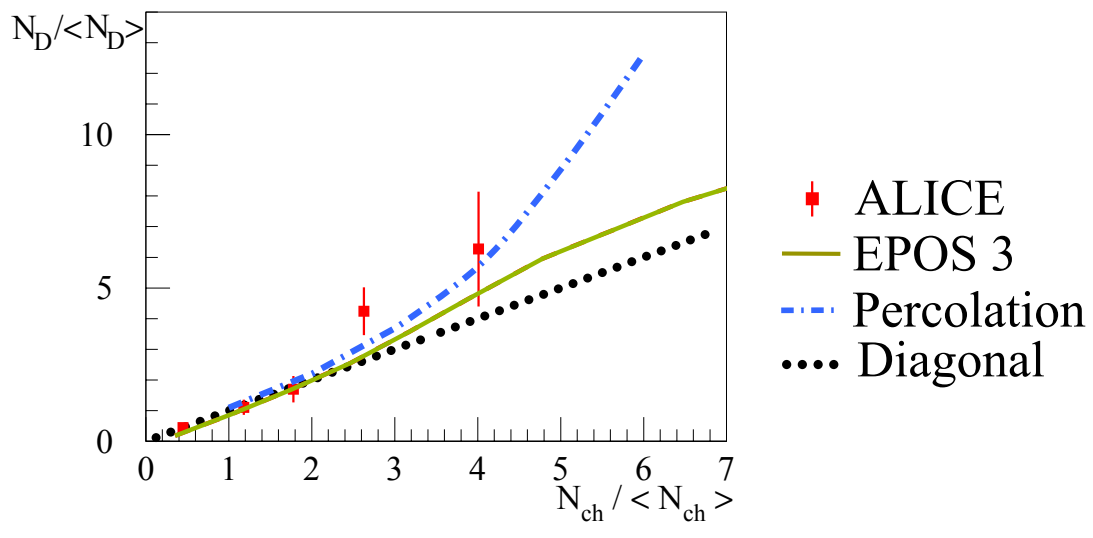

Figure 1. Average D-meson relative yield for $1<p_{t}<2 \mathrm{GeV}$ as a function of the relative charged multiplicity in pp collisions at $\sqrt{s}=7 \mathrm{TeV}$. Experimental data [3] compared to EPOS 3 [4] and string percolation model [5].

Interaction probability amplitude of two dipoles with transverse coordinates $\left(\mathbf{r}_{1} \mathbf{r}_{1}^{\prime}\right)$ and $\left(\mathbf{r}_{2} \mathbf{r}_{2}^{\prime}\right)$ is given by $[6,7]$ :

$$
f=\frac{\alpha_{S}^{2}}{8} \ln ^{2} \frac{\left(\mathbf{r}_{1}-\mathbf{r}_{1}^{\prime}\right)^{2}\left(\mathbf{r}_{2}-\mathbf{r}_{2}^{\prime}\right)^{2}}{\left(\mathbf{r}_{1}-\mathbf{r}_{2}^{\prime}\right)^{2}\left(\mathbf{r}_{2}-\mathbf{r}_{1}^{\prime}\right)^{2}},
$$

Note, that two dipoles interact more probably, if their ends are close to each other, and (others equal) if they are wide. The model also incorporates confinement effects.

The charged multiplicity is calculated in the approach of colour strings, taking into account their finite rapidity length and interactions due to non-zero transverse radius of string $r_{\text {str }}-$ string fusion $[8,9]$. Mean multiplicity of charged particles originated from $k$ overlapping strings is modified as $\langle\mu\rangle_{k}=\mu_{0} \sqrt{k}$, where $\mu_{0}$ is a mean charged multiplicity from one single string.

Parameters of the model are fixed from the pp, $\mathrm{p}-\mathrm{Pb}$ and $\mathrm{Pb}-\mathrm{Pb}$ data on the total inelastic cross section and charged multiplicity in wide energy range [10].

We note, that contrary to the total charged particle multiplicity, which is influenced by string fusion process, the heavy flavour yield is assumed to be proportional to the number of initial strings.

\section{Results and discussion}

The results of the calculation are shown in Figure 2.

The results demonstrate that reduction of total charged multiplicity due to string fusion gives faster-than-linear behaviour of relative charmed meson yield, observed in the experiment.

At largest multiplicities, however, another mechanism of charm enhancement may play an important role, which is related to Schwinger (or thermal-like) production of c- $\bar{c}$ pairs [11]. We suppose that the effect may be more pronounced also in $\mathrm{p}-\mathrm{Pb}$ collisions at LHC energy, where the string density is larger, and allowing to produce charm quarks by strings of high string tension.

These two mechanisms of the charm growth could be discriminated in the experiment by studying the long-range correlations of the relative $\mathrm{D}$ meson yield in one rapidity interval with the average $p_{t}$ or relative strangeness in another window $[12,13]$. The hard mechanism of the charm production 


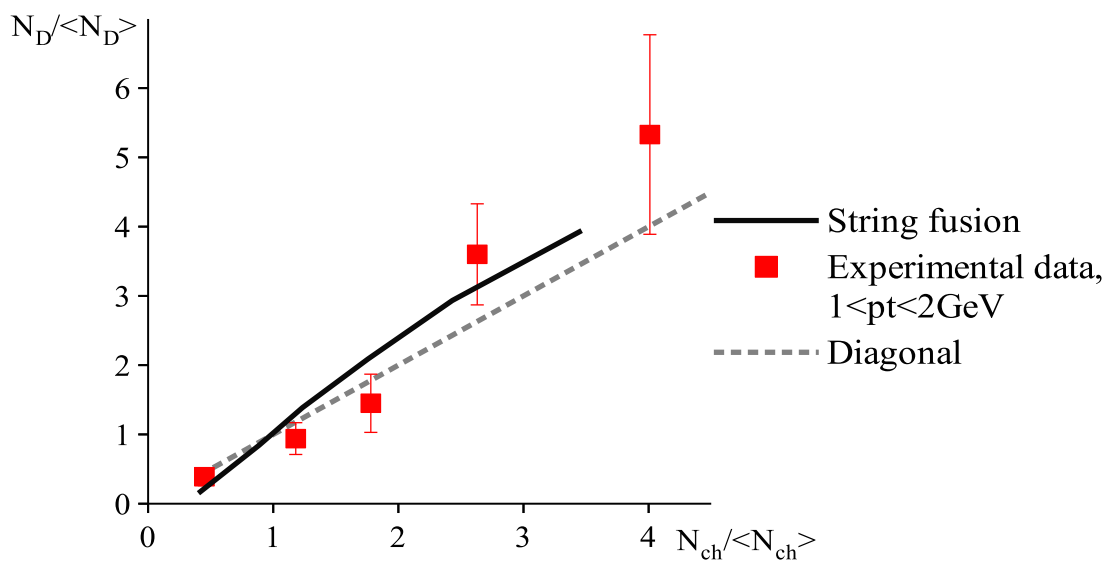

Figure 2. Relative average D-meson yield as a function of charged multiplicity, calculated in MC string fusion model, compared to experimental data [3] for pp collisions at $\sqrt{s}=7 \mathrm{TeV}$.

will contribute to the negative correlation, and the one related to the string tension increase will give positive correlation.

\section{Acknowledgments}

The research was funded by the grant of the Russian Science Foundation (project 16-12-10176).

\section{References}

[1] V. N. Kovalenko, Phys. Atom. Nucl. 76, 1189 (2013), arXiv:1211.6209 [hep-ph].

[2] V. Kovalenko, V. Vechernin, PoS (Baldin ISHEPP XXI) 077, arXiv:1212.2590 [nucl-th], 2012.

[3] J. Adam, et al. (ALICE Collaboration), JHEP 09, 148 (2015), arXiv:1505.00664 [nucl-ex].

[4] K. Werner, B. Guiot, Iu. Karpenko, T. Pierog, G. Sophys, DESY Conf. Proc. 2016-01, p. 66-70, arXiv:1602.03414 [nucl-th] (2016)

[5] E. G. Ferreiro and C. Pajares, arXiv:1501.03381 [hep-ph] (2015).

[6] C. Flensburg, G. Gustafson, L. Lonnblad, Eur. Phys. J. C 60, 233 (2009).

[7] G. Gustafson, Acta Phys. Polon. B 40, 1981 (2009), arXiv:0905.2492 [hep-ph].

[8] M. A. Braun, C. Pajares, V. V. Vehernin, Phys. Lett. B 493, 54 (2000).

[9] M. Braun, R. Kolevatov, C. Pajares, V. Vehernin, Eur. Phys. J. C 32, 535 (2004).

[10] V. Kovalenko, PoS (QFTHEP 2013) 052 (2013).

[11] J. Schwinger, Phys. Rev. 82, 664 (1951); T. S. Biro, H. B. Nielsen, and J. Knoll, Nucl. Phys. B. 245, 449 (1984).

[12] V. Kovalenko, V. Vechernin, J. Phys. Conf. Ser. 668, 012065 (2016), arXiv:1509.06696 [hep-ph].

[13] V. N. Kovalenko, A. M. Puchkov, G. A. Feofilov, Bull. Russ. Ac. Sc. Phys. 80, 966 (2016) preprint http://hdl.handle.net/11701/1743 . 